\title{
LA NUEVA ECONOMÍA Y SUS FORMAS EMPRESARIALES
}

José Domingo Begazo*

jbegazov@unmsm.edu.pe

\section{RESUMEN}

La globalización de la economía -como resultado de los avances logrados por la electrónica y las telecomunicaciones, y la liberalización de los mercados- ha creado por primera vez en la historia un único modelo económico mundial, en el que la innovación es cada vez más importante y el trabajo intelectual desplaza al físico, dentro de un mundo esencialmente inestable y en proceso de cambio continuo.

Palabras Claves: Sociedad Cognomática; Nueva Economía; Nasdaq; Stakeholders; Internet; Virtualidad.

\section{ABSTRACT}

Economics globalization -as a result of the development achieved both in electronics and telecommunication and market freedom- has createde for once in history a new economics model, where innovation is more important than ever thus intellectual work substitutes its physical counterpart inside the dynamics of an unstable and countinous changing process world.

Key words: Economic globalization, Market freedom, new economics model.

\section{ANTECEDENTES}

\section{En la sociedad industrial}

Antiguamente podíamos definir que la empresa más grande se comía a la empresa más chica; en el mundo de los bits, podemos definir que el pez más rápido se come al más lento.
Cifras recientes de la Organización para la Cooperación y el Desarrollo Económico (OCDE) nos confirman que las economías desarrolladas están atravesando por uno de los auges económicos más importantes de los últimos treinta años. Se trata de una revolución tecnológica de la cual somos conscientes desde que se inició el

* Doctor en Administración. Magíster en Administración y en Desarrollo Económico Social. Profesor Principal y Miembro Permanente de la Unidad de Investigación de la Facultad de Ciencias Administrativas de la Universidad Nacional Mayor de San Marcos. Profesor de posgrado en diferentes Universidades y Centros Superiores. Participante y Ponente en los últimos seis Congresos Nacionales de Filosofía, y Conferencista invitado en diversos eventos realizados fuera del país. 
desarrollo de la electricidad, de la electrónica y de la informática. Todo el nuevo mercado de alta competencia se mueve por la lógica de Internet y el comercio electrónico.

\section{La globalización y el pensamiento económico}

La globalización de la economía, como resultado de los avances logrados por la electrónica y las telecomunicaciones, y la liberalización de los mercados, ha generado un único modelo económico mundial donde la innovación resulta importante al igual que el trabajo intelectual.

Aunque quizás todavía sea pronto para disponer de un completo cuerpo doctrinal de la Nueva Economía (NE), ya comenzamos a contar con suficientes elementos de juicio para moldear su perfil y definir sus principales características.

La NE -como consecuencia de la velocidad de los cambios tecnológicos y regulatorios que la han hecho posible- ha madurado como realidad mucho antes de que ningún economista haya advertido y teorizado completa y convincentemente acerca de ella.

En la historia del pensamiento económico podríamos convenir que Adam Smith ha renovado su pensamiento teórico cuando sostenía que la riqueza procedía del trabajo, la cual se incrementa debido a que aumenta la destreza de la fuerza de trabajo, ahorra tiempo y permite el empleo de ingenios mecánicos.

Este autor enfoca el concepto desde las siguientes preguntas:

- ¿El valor de una mercadería debe ser medido por la cantidad de trabajo que su producción ha exigido al productor-vendedor? En este caso el valor tendría por medida el trabajo gastado incorporado en la mercadería (labour embodied).

- ¿O por la cantidad de trabajo que el consumidor-comprador hubiera debido realizar para fabricarla? El trabajo ahorrado (labour saved).

- ¿O por la cantidad de trabajo que esta mercadería permite comprar, encomendar a otros? El trabajo encomendado (labour commanded). Y Smith se inclina hacia la tesis del trabajo encomendado.
Del análisis del pensamiento primigenio de A. Smith podemos colegir que el concepto del trabajador directo que conllevó a toda una fundamentación del pensamiento económico en la Sociedad Industrial, ha derivado al análisis del trabajador indirecto en este tipo de Sociedad Cognomática.

Joseph A. Schumpeter puede ser considerado como el precursor de la nueva economía, ya que analizó el sistema capitalista y sostuvo la teoría de que las innovaciones realizadas por los empresarios son el factor estratégico del desarrollo económico y ocupan una posición central en el proceso del ciclo económico. Para Schumpeter, el régimen del capitalismo es el régimen del progreso; genera continuamente innovaciones y se sostiene en ellas. Su original y ahora clásico concepto de la "creación destructiva" fue el primer alegato teórico acerca de la influencia de la innovación en el proceso económico, hoy en día eje central de la realidad económica que nos envuelve. Según él, tales innovaciones "revolucionan constantemente desde adentro la estructura económica, destruyendo sus elementos anticuados y creando otros nuevos".

Llama la atención la vigencia de pensamientos económicos concebidos hace más de 200 años (en el caso de Adam Smith), y sesenta años (en el caso de J. Schumpeter), y la extrema fragilidad de cierto pensamiento contemporáneo representado por el Club de Roma y el premio Nóbel Lester Thurow, que sostenían lo contrario de lo que ha terminado por acontecer.

Las teorías agoreras de la economía que han venido vaticinando los límites del crecimiento han sido desmentidas por la realidad, gracias a la maravillosa capacidad creativa del ser humano, que con sus invenciones sigue empujando el mundo hacia adelante.

En la medida en que el cambio tecnológico fue lento o muy lento el modelo sirvió, pero cuando la innovación tecnológica entró en escena, no como un sujeto marginal de la obra económica sino como actor principal del cambio y el desarrollo económico, el modelo quedó obsoleto.

La teoría económica, que utilizó hasta ahora un aparato matemático muy limitado y permaneció alejada de las ciencias llamadas duras, busca ahora la convergencia y el auxilio de otras 
teorías con las cuales ir conformando un nuevo modelo de renovada capacidad explicativa.

Resumiendo: los modelos económicos que revolucionaron el ámbito de la empresa, hoy deben ser reconsiderados a la luz del mundo del tercer milenio, donde conceptos como globalización y tecnologías de la información se ven afectados por el avance arrollador de Internet, el impulso de los mercados financieros y una dinámica humana distinta, que marcan el paso de una nueva sociedad.

Adicionalmente la innovación es cada vez más importante y el trabajo intelectual desplaza al físico, dentro de un mundo esencialmente inestable y en proceso de cambio continuo, lo que ha generado nuevas ciencias, ramas del conocimiento, formas de comunicación social, materiales, formas de capital, actividades económicas, mercados, formas de organizar y realizar la producción, formas para distribuir los productos y servicios, formas de compra-venta, formas de consumo, formas de participar en los resultados de la actividad económica, formas de crecimiento económico, así como nuevas formas de transmisión y acceso a la información y el conocimiento.

\section{INTRODUCCIÓN}

Muchas cosas están cambiando hoy día en el mundo de los negocios y en el conjunto de la sociedad. La Nueva Economía (NE) y sus elementos más representativos (la tecnología y la globalización), están modificando hábitos y comportamientos sociales e individuales.

La NE se comienza a desarrollar en la escena económica durante la segunda mitad de los años noventa, particularmente en los Estados Unidos, con la combinación de los siguientes factores:

- El supuesto fin del ciclo económico, es decir, de las expansiones y las recesiones económicas. Podemos analizar en este punto el pensamiento de N. D. Kondratiev que indicaba que las economías capitalistas evolucionaban en ciclos de cincuenta años, con un primer período de expansión y otro segundo de contracción, cada uno de veinticinco años.

- La mayor disponibilidad de capitales para financiar proyectos empresariales en un mundo con mercados de capitales globales y;
- La emergencia de Internet y las nuevas tecnologías de la información.

Todo ello generó un aumento de la productividad y una mayor disponibilidad de recursos financieros (especialmente del llamado capital de riesgo).

La desregulacion financiera en los años ochenta y noventa permitió una mayor expansión internacional de las entidades financieras y de los mercados de valores, así como la entrada de nuevos competidores en la financiación de empresas y la colocación de ahorro por parte de las familias. El crecimiento espectacular de las bolsas y de los mercados de deuda en los años noventa permitió que la nueva financiación dirigida a las empresas aumentara vertiginosamente.

Si se tuviera que establecer la fecha de nacimiento de la NE, ésta sería el 27 de octubre de 1986, día en que las Bolsas de Londres y New York decidieron abolir las comisiones fijas en la compraventa de acciones, lo que vino a ser llamado entonces el Big Bang, porque puso fin a años de transacciones tradicionales a favor de una completa operación electrónica de dichos mercados. El espectacular crecimiento de los mercados financieros globales ha sido el resultado del desarrollo de los mercados electrónicos, y desde entonces la desregularización del comercio ha hecho que los mercados sean más eficientes, fluidos y populares en todo el mundo.

\section{¿QUÉ ES LA NUEVA ECONOMÍA?'}

- Primera definición. Es una revolución de la productividad, un proceso que permite a las compañías incrementar la productividad mediante el uso de la tecnología, de tal forma que la economía pueda crecer más rápidamente sin inflación.

- Segunda definición. Estamos hablando de un mundo en el que la gente trabaja con sus cerebros en lugar de hacerlo con las manos; un mundo en el cual las Tecnologías de la Información (TI) crean la competencia global; un mundo en el que la innovación es más importante que la producción en masa; un mundo en el que la inversión compra nuevos conceptos o los medios para crearlos, en lugar de comprar nuevas máquinas; un mundo en el cual el rápido cambio es una constante; 
finalmente, un mundo que por su emergencia es tan diferente que sólo puede ser descrito como una revolución ${ }^{2}$.

- Tercera definición. Concepto inspirado en el crecimiento norteamericano y relacionado con las nuevas tecnologías, que contradice las reglas habituales debido a su duración, ausencia de inflación de precios y descontrol salarial, productividad ascendente y capacidad de producción que siempre excede a la demanda, etc.

En resumen, podemos decir que la Nueva Economía está formada por actividades directamente conectadas con la electrónica, la informática, la multimedia, Internet y la biotecnología. En una acepción más amplia, la NE también se extiende a las actividades clásicas que son poco a poco penetradas, reestructuradas y reorganizadas por las nuevas tecnologías. En los últimos años, el mundo ha emprendido la transición de una economía industrial a otra basada en la información. El auge del correo electrónico e Internet configuran la infraestructura básica de la NE.

Estamos en los albores de la NE cuyo corazón es el microprocesador que viene de la mano con internet; economía en red en contraposición de la economía de escala, cuya principal característica es la reducción de costos, mientras que la economía en red es aquella que surge cuando se van agregando agentes a una comunidad. El poder se basa cada vez menos en parámetros físicos y empieza a ser definido como la capacidad de almacenar, gestionar, distribuir y crear información.

La NE no sólo está conformada por las empresas puramente virtuales; en realidad, este proceso ha incorporado a las compañías de la economía tradicional que han lanzado sus versiones digitales. Una de las áreas más dinámicas en este desarrollo es la industria bancaria que ha hecho importantes inversiones en tecnología, los que les permite reducción de costos, ahorro de tiempo, descongestionamiento de las agencias, atención las 24 horas del día, así como la búsqueda de la fidelización del usuario. razones:

El auge de la NE se debe a las siguientes

- Uso intensivo de las Tecnologías de la Información. Con ellas se acortan los tiempos de las transacciones económicas y la toma de decisiones; el uso de la alta tecnología dentro de las empresas ha sido un importante factor de apalancamiento.

- Preponderancia de las Empresas Tecnológicas. Las empresas fueron derivando de industrias intensivas en mano de obra hacia industrias de alta tecnología y servicios menos intensivos en mano de obra.

- Incremento de la Competitividad. Con este aumento, los países y las organizaciones pueden abarcar cada vez más mercados con productos más eficientes, a menores precios.

- Desarrollo del Capital Humano. Las inversiones de los últimos años destinadas a promover el desarrollo humano y a conocer las relaciones humanas han sido muy importantes. Se dieron en todo ámbito: técnico, profesional y de alta especialización.

Durante la celebración de la Microsoft Government Leaders Conference realizada en Seattle, Estados Unidos, en abril del 2002, se presentó el estudio "The IT Economic Impact on Local Economies”, que revela los beneficios más importantes que representan las Tecnologías de la Información para la continuidad y el éxito de las economías locales, en 28 países del mundo.

La investigación proveyó análisis y predicciones acerca de las inversiones en TI, la creación de puestos laborales, los ingresos por impuestos y la formación de compañías como resultado de las inversiones tecnológicas. Los países analizados incluyen 14 economías emergentes y 14 desarrolladas, que toman en cuenta más del $90 \%$ de las inversiones tecnológicas realizadas en todo el mundo en el año 2001. Una de sus principales conclusiones fue que la industria tecnológica generalmente incide en el crecimiento del PBI de un país entre 2 y 5 puntos, al igual que el empleo tecnológico que creció más rápidamente que en otros países.

\section{LA MAGNITUD DEL CAMBIO}

La imprenta generó la era de Gutemberg hace 500 años; el motor eléctrico, creado en 1888, no aumentó mucho la productividad sino hasta 40 años después de inventado. Tomó todo ese tiempo para que los industriales uniformaran el motor, reconstruyeran las fábricas en torno a él y lo conectaran a redes de electricidad. 
La industria del automóvil nació en 1906; para 1908 habían 240 firmas en el negocio y después de la crisis de 1910 sólo quedaron dos: Ford y General Motors. Ahora los cambios son más rápidos y se utiliza menos fuerza; aumenta la confiabilidad, y lo más importante, disminuyen los precios.

Los chips están presentes en todos los aspectos de la actividad humana: en los celulares, computadoras, agendas, artefactos electrodomésticos, juguetes, siderúrgicas, oficinas inteligentes, etc. Desde la década del 40 la revolución cibernética viene dando pasos agigantados, mientras que la web no llega a siete años pero ambas han generado un mercado electrónico que crece rápidamente y que ha creado una generación de compañías “.com”. Podemos decir entonces que las compañías de la NE se caracterizan por tener velocidad, innovación y disposición para arriesgarse, y se guían por tres reglas básicas: la constante innovación que origina nuevas TI, el uso de tecnología que aumenta la productividad, y el incremento del crecimiento económico.

Frente a los economistas tradicionales que concebían el crecimiento como el producto de la fuerza laboral y el capital físico, la nueva teoría del crecimiento económico que iniciara el premio Nobel (1987) Robert Solow y actualmente desarrolla el profesor de la Universidad de Stanford, Paul Romer, indica que "la inversión de capital no es en absoluto el factor clave del crecimiento económico. Tampoco lo es el aumento de trabajadores". En cambio, se encuentra presente un factor residual, una categoría indefinida y amplia, que se conoce ahora como innovación o tecnología, señalándose la importancia de la educación como promotora del conocimiento y la inventiva. Ideas como éstas han llevado a debates más amplios sobre el valor del "Capital Humano".

Desde este punto de vista, la innovación, es la única fuente de crecimiento sostenible, y la rápida difusión de las tecnologías de la información, una garantía para el desarrollo de las ventajas competitivas.

La famosa ley de Moore, según la cual cada 18 meses se duplica la potencia de los microprocesadores, es la palanca que mueve hacia delante la "Nueva Economía".

El capital, tradicionalmente considerado como un valor (en forma de dinero o máquinas), que puede ser utilizado para crear más valor, toma forma hoy de conocimiento, patentes, propiedad intelectual, bases de datos y software, incluida la todavía vaga idea de capital social que tiene que ver con la capacitación y confianza personal de los agentes que operan en el mercado.

Las nuevas circunstancias han creado también un nuevo concepto que cabría llamar la Economía del Tiempo, dada por la sociedad red, que, según sostiene Manuel Castells ${ }^{3}$, difunde esta nueva forma de capital y con ella la posibilidad de sustentar el crecimiento económico.

Ser el primero en el mercado ofrece ventajas en la economía de la información: los mercados basados en bits que se mueven a la velocidad de la luz tienden a recompensar la cualidad frente a la cantidad. Tal y como el físico Freeman Dyson ha observado "no hay que sacrificar economías de tiempo por economías de escala”.

La “NE” es esencialmente paradójica. Si los mercados conocen toda la información disponible, pueden perfectamente deducir sus consecuencias y reaccionar tan pronto como sea posible a cada novedad. Pero si nadie obtiene información, entonces los mercados no serán perfectamente informados.

En realidad no podemos conocer todo, o al menos, no podemos pretender que lo conocemos todo. De ahí la paradoja de Grossman, según la cual siempre habrá un mercado para la información.

La "NE" es un concepto acaparador de multitud de síntomas sociales y económicos y ha traído consigo o ha acogido otros conceptos mas escuetos como Capital Intelectual y Capital Social que llevan el fenómeno de la gestión empresarial de moda: la creación de valor económico y la lucha por la creación de riqueza.

Comencemos por indicar que la creación de valor es un concepto asociado, en su origen, al mundo anglosajón, siendo A. Rappaport el autor creador de esta teoría y que más ha impulsado la misma. Él habla del "shareholder value creation" asociado al capitalismo más duro. Las empresas suavizando el término hablan del "stakeholder value creation" o "economic value creation".

Grandes empresas del mundo afirman haber implementado la gestión basada en el valor, 
"value-based management" que es un sistema de gestión que pretende hacer del individuo parte esencial de la estrategia de la empresa y cuya finalidad es la generación de valor a largo plazo.

Es por ello que los modelos desarrollados con este fin tales como EVA (Gestión basada en la creación de Valor o Value Based Management VBM), CFROI, etc., no pueden basarse exclusivamente en medidas financieras, especialmente a corto plazo, sino que toman en consideración el capital intelectual de la empresa, es decir las relaciones estratégicas con proveedores y clientes, la eficiencia de los procesos internos, los sistemas de calidad, los procesos de I\&D, los medios tecnológicos, etc., como generadores de valor, lo que requiere evaluarlos adecuadamente.

Una razón por la que la "NE" ha sido llamada "invisible" es que los antiguos métodos de contabilidad tienen problemas para detectarla.

Para eso el Balanced Scorecard, traducido el español como Cuadro de Mando Integral y Tablero de Comando, tiene que ver con un sistema de información y evaluación interna y externa de la empresa encaminado al diseño de estrategias y objetivos empresariales y la elaboración de indicadores y factores claves capaces de medir la gestión y con ella la creación de valor.

La evolución de la economía de los negocios también exige de una evolución de la propia concepción de los servicios de "outsourcing". La viabilidad de la utilización del "outsourcing" como herramienta de gestión para el desarrollo de la "NE" no está limitada ni por sectores ni por el tamaño de las empresas.

En el mundo de la economía digital el papel del socio tecnológico está mucho más próximo al negocio fundamental del cliente, por lo que se le exige una mayor injerencia en el mismo y la aportación de más valor añadido. Así se establece una verdadera relación de alianza o "partnership" en la que se comparten los objetivos, riesgos y beneficios asociados a la colaboración.

Pocos problemas son más importantes y desconcertantes en una compañía que transformar una cultura empresarial de la Vieja Economía para estar a la altura de las necesidades de la Nueva Economía donde el tiempo es la fuente más preciada y la velocidad el atributo más valioso en un mundo que va al ritmo de la web.

\section{1. ¿Cuánto durará?}

Es difícil pronosticar el periodo de vida de este modelo y saber si será prolongado o no, pues los cambios son cada vez más rápidos y las empresas más flexibles, y de aquí al futuro los retos serán otros.

La NE y la VE no sólo se parecen sino que se necesitan mutuamente, es decir, se complementan; no es realista esperar que con el tiempo las empresas virtuales reemplacen a las convencionales ${ }^{5}$.

Más bien unas van a tomar las cualidades de la otra. Así Amazon.com está construyendo grandes almacenes al más puro estilo de las cadenas de tiendas de Estados Unidos; por su parte, las cadenas de tiendas construyen sus sitios web para poder ofrecer sus productos tal y como lo hace Amazon.com. Ese es el futuro: la convergencia.

La convergencia digital es la quinta esencia de la NE; sistemas tecnológicos y económicos desagregados hoy forman parte de un nuevo hipersistema de información que integra medios y contenidos de la más variada especie donde la distribución de la inteligencia a través de las redes hace que las decisiones se tomen allá donde se encuentra la información.

La NE es más fluida, la apertura de los mercados y la nueva sociedad red relativizan el papel del mercado que tiende a convertirse en un factor más de la producción, acerca del cual los agentes económicos actúan según su conveniencia.

Las peculiaridades de la economía digital impulsan a las compañías, casi de manera inevitable a buscar escala masiva. En lugar de los "retornos decrecientes a escala" que según los textos evitan que las empresas crezcan demasiado, la NE se caracteriza por los retornos crecientes a escala.

\section{AMÉRICA LATINA FRENTE A LA NE}

El Banco Interamericano de Desarrollo (BID) ha elaborado un documento denominado "Desarrollo más allá de la Economía”, donde indica que América Latina ha ingresado a este milenio con un desempeño económico modesto y profundamente desigual e inestable. 
Entre los factores estructurales que separan a América Latina de otras regiones y a los países latinoamericanos entre sí, podemos mencionar:

1. Demografía: la estructura de edades de la población y sus cambios, control de la natalidad.

2. Geográficas: tanto naturales como creadas, y su influencia que puedan ejercer en las posibilidades económicas. Factores como la productividad de las tierras, las condiciones de salud debido al clima y el acceso a los mercados.

Al igual que la demografía, la geografía no es una constante a través del tiempo, las condiciones naturales, tales como la ubicación y el clima o el acceso al mar pueden no modificarse mayormente pero su influencia sobre los resultados del desarrollo puede alterarse radicalmente dependiendo de la localización de la población y las actividades económicas, las inversiones en infraestructura y servicios públicos y los cambios en los costos y métodos de transporte entre otros factores.

3. Las instituciones: se requieren de buenas instituciones para acelerar el desarrollo, la cuestión es entender las razones por las cuales las instituciones son como son y los factores que impiden mejorarlos.

Las compañías de la Antigua Economía están condenadas a un crecimiento lento a la inversa de los inversionistas en la NE que tienen un alto rendimiento en la especulación del desarrollo tecnológico de las empresas de la $\mathrm{NE}^{6}$.

Internet está cambiando la sociedad, la está convirtiendo en una nación sin fronteras, cuyos integrantes son libres de producir información y de obtenerla, donde las distancias están expresadas en segundos. Donde el único requisito es tener acceso a una computadora y donde la democracia ha encontrado un nuevo camino de renovación.

La transición a la Nueva Economía del conocimiento está provocando cambios sociales, culturales, morales, institucionales y políticos.

Esta es la nación digital, una comunidad de individuos alrededor del mundo que están conectados unos a otros por medio de sus computadoras personales, compartiendo no sólo sus propias vivencias a través del correo electrónico, sino además usufructuando información de toda clase.

\section{CONCLUSIONES}

1. La Nueva Economía es una economía en tiempo real. Y ésta ha entrado en una nueva fase de cambios desconcertantes lo que hace que los inversionistas estén cada vez menos seguros sobre cuáles serán los ganadores en el futuro. Lo que le importa al mercado son las ganancias.

2. La NE llamada también sector ".com» está ligada a la revolución digital y la competencia es global, el capital es abundante, el desarrollo dinámico y, sobre todo, a la gente le gusta cambiar de trabajo, pero la contribución de la tecnología al empleo es mínima.

3. El nuevo comercio y el dinero electrónico eliminarán muchos intermediarios, pero la eliminación de estos trabajos irá de la mano de la creación de otros, asociada a los nuevos procesos "on line".

4. A esto contribuyen los ejecutivos .com que son la clave del éxito de las empresas de la $\mathrm{NE}$; con el reclutamiento del "e-people" o la nueva generación: jóvenes, informales, flexibles, dinámicos (Prozac) y ambiciosos ejecutivos, que visualizan las inversiones en el sector tecnológico y de comunicaciones.

5. Existe la idea de que todo lo que tenga que ver con la NE es buena inversión y todas las compañías de la NE están condenadas.

"Son las empresas dedicadas a la venta de tangibles: ladrillos, alfombras, pinturas, botas, acero y productos químicos etc. o la construcción en sectores tan tradicionales como ferrocarriles, automóviles y construcción de buques entre otros"4.

En el Perú, si en la Sociedad Industrial podríamos jactarnos de nuestras riquezas naturales y era válido el pensamiento de A. Raymondi: "El Perú es un mendigo sentado en un banco de oro". Ahora en esta Sociedad del Conocimiento donde ya no podemos hablar del valor-trabajo sino del valor-conocimiento es necesario e imprescindible que nuestra mejor riqueza, sea nuestra juventud preparada para asumir los nuevos retos. Y entonces debemos ser capaces de desarrollar, diseñar e implementar planes de manera rápida que es la condición del éxito. 


\section{Notas}

1 Gèlinier, Octave y Patearon, Emmanuel. La Nueva Economía del siglo XXI.

2 Autores. Encyclopedia of the New Economy.

3 Manuel Castells, La era de la Informática.

5 Kelly, Kevin. New Rules for the New Economy.

${ }^{6}$ Jonas Ridderstràle y Kjell Nordstrom. Funky Business.

\section{BIBLIOGRAFÍA}

Álvarez Roldán, Roberto (2000). E. change, el lado humano de la Economía digital. Edit. Gránica.

Artículos diversos en revistas y periódicos especializados.

Castells, Manuel (1998), La era de la informática. Volumen 1, Alianza Editorial.

Kelly, Kevin (1998). New rules for the New Economy, New York, Penguin Books.
Gélinier, Octave y Patearon, Emmanuel (2001). La nueva economía del siglo XXI, Edit. Paidós.

Grove, Andrew (1996). Only the paranoid survive. New York, Harper Collins.

Ferreira Mendonca y Darlan Sebastiäo (2000), Creación de futuros, Edit. Brasil.

Ridderstrále, Jonas y Norström, Kjell (2000), Funky business. Edit. Prentice Hall.

Science Vol. 238, núm. 4828, págs. 754-756 del 6 de noviembre de 1987.

Suárez Suárez, Andrés S. (2001). Nueva economía y nueva sociedad. Edit. Prentice Hall.

Tapscott, Don (2000). La creación de valor en la Economía digital. Edit. Gránica. 